\title{
Experimental verification of visual method for measuring displacements of contact line elements
}

\author{
Jacek Skibicki ${ }^{1, *}$ \\ ${ }^{1}$ Gdansk University of Technology, Institute of Electrical Engineering, Division of Electrical Engineering in Transport, \\ ul. Narutowicza 11/12, 80-233, Gdańsk, Poland
}

\begin{abstract}
The increase of rail vehicles speed, as well as the increase of their power, puts high demands on the power delivery system for traction vehicles The most critical point in the vehicle's power supply circuit is the contact between the current collector and contact wires. Ensuring a reliable co-operation of the current collector and contact line, requires technical development of the contact line and current collector construction, as well as improvement of diagnostic systems of these components. This is translated into the need to develop new measurement methods, which allow for more complete definition of the parameters of these elements of traction vehicle power supply system. Each new measurement method requires verification, performed by calibrating or comparing the results with another measurement method which has already been tested. The article presents results of experimental verification of a visual method for measuring the displacement of contact wires, with a proven method based on short range laser rangefinders and calliper as reference measurement.
\end{abstract}

\section{Introduction}

Diagnostics of elements of the traction vehicles power supply can be divided into diagnostics of the contact line itself, diagnostics of current collectors and monitoring of proper cooperation of these elements. Diagnostic stands of the contact line are constructed in the form of mobile

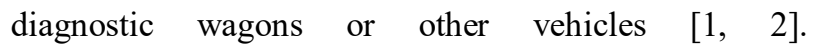
Measurements of contact line parameters are often performed by using special measurement current collectors [1]; visual, contact-less methods, where the measuring system does not affect the contact line are also known [3-6]; sometimes measurements performed with the use of a collector are supplemented and supported by visual techniques [7, 8]. In contrast, diagnostics of current collectors is usually performed with the use of stationary diagnostic stands, where static characteristics of the collector and suspension of the slipper are tested [9]. Evaluation of the technical condition of strips is carried out using 3D visualization devices [10-13]. On the other hand, correctness of cooperation between the collector and the contact line can be monitored with the use of stationary measurement points which help to determine the correctness of the collector's adjustments based on displacements of the contact line caused by its impact when a vehicle passes through the diagnostic stand. In order to measure contact line displacements, short range laser rangefinders or other optical devices are most commonly used. They make it possible to evaluate movements of the contact wire in the vertical axis $[14,15]$.
The proposed method based on a stationary 2D camera and advanced image analysis allows for the measurement of the position (displacement) of contact wires in a contact line, or of other elements, in a twodimensional plane. For monitoring the cooperation of the current collector and the contact line, measuring displacements of contact wires in a two-dimensional plane gives more possibilities, compared to the existing stands, which allow only for measurements in the vertical axis. In addition to determining the condition of adjustment of the collector's clamping force, it is possible to evaluate, to some extent, the technical condition of the slipper strip.

Each new measurement method requires verification, performed by calibrating or comparing the results with another measurement method which has already been tested. This article presents the results of experimental verification of the above-mentioned measurement method for displacements of contact wires in the catenary network with a verified method using short range laser rangefinders.

\section{Subject of measurement}

The subject of measurement is the displacement of the contact wire of contact line, caused by external force coming from the current collector or from a different source. The displacement range determined on the basis of previous measurements was set at $\pm 10 \mathrm{~cm}$ in both axes, with the possibility of exceeding it by $2 \mathrm{~cm}$ in each direction, to allow for recording of displacements deviating from the typical ones. Frequency of oscillation

\footnotetext{
* Corresponding author: jacek.skibicki@pg.edu.pl
} 
courses of the contact line does not usually exceed a few $\mathrm{Hz}$ [14]. To represent the course of changes correctly, it is assumed that the sampling rate of the signal should not be less than $100 \mathrm{~Hz}$.

\section{Methodology of measurement}

In the first stage, comparative measurements of oscillations of the contact wire of contact line caused by external force were performed with the use of a laboratory test stand, whose diagram is shown in Fig. 1.

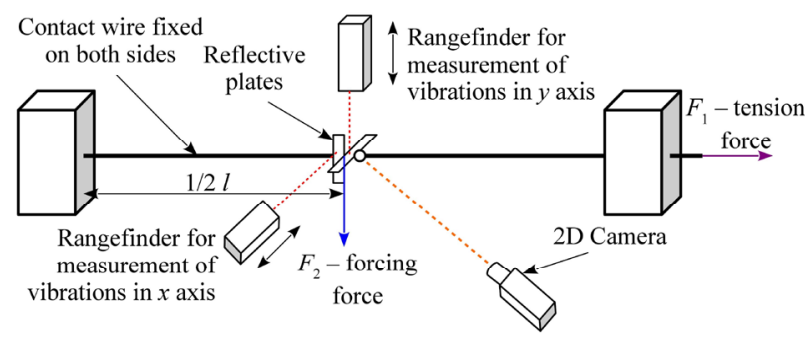

Fig. 1. Diagram of stand used for comparing results obtained with the use of two measurement methods.

A section of typical contact wire type Djp100 used by the PKP, with the length of $l=28.8 \mathrm{~m}$, was fixed on both sides and tensioned with the force of $F_{1} \approx 10 \mathrm{kN}$. Mid-way along the length $l$, the forcing force $F_{2}$ was applied and, after its peak removal, oscillatory displacement of the contact wire was recorded using both measurement methods at the same time.

The second stage of the comparison was to test which method gives more accurate results, i.e. the results with a lower level of measurement uncertainty. For this purpose, measurements were performed at the stand presented in Fig. 2.

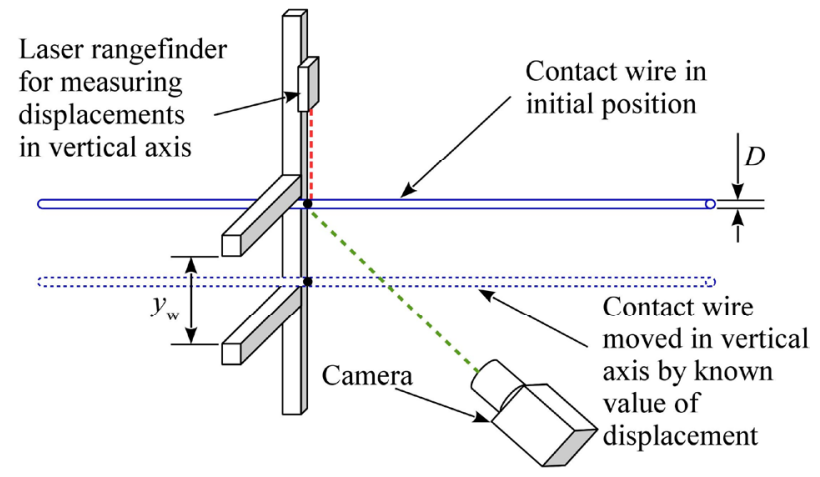

Fig. 2. Stand used for comparing accuracy of measurement methods.

The contact wire was moved by a known value in the vertical axis, and this displacement was recorded using a laser sensor and the camera system. The results were compared by way of a reference measurement performed with a calliper. In all the cases, the measurement uncertainty analysis was conducted, and all the indirect measurements were made with the use of high quality laboratory instruments. Visual measurements were made for the configuration of the stand where the uncertainty analysis showed that the highest measurement accuracy would be achieved.

Detailed information on each measurement is given below.

\section{Measurements performed with the use of laser rangefinders}

The measurements were performed with the use of short range laser rangefinders. Due to torsional fluctuations of the contact wire, it was necessary to use a third rangefinder to compensate for those fluctuations. The principle of compensation is presented in Fig. 3.

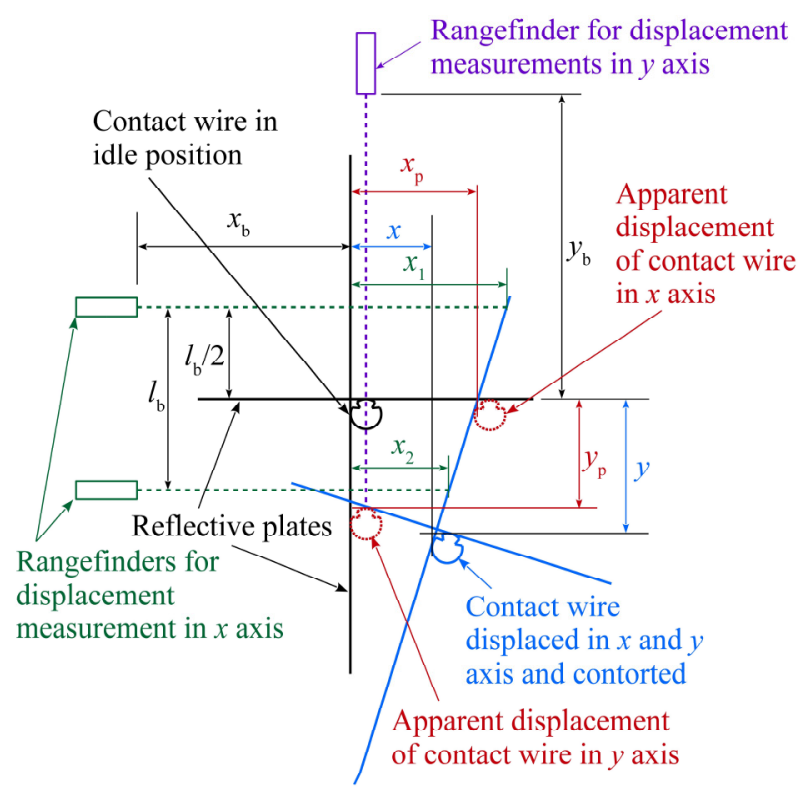

Fig. 3. Compensation of torsional vibrations with the use of a third laser rangefinder.

In the system presented in Fig. 3., in the idle state, the contact wire location is determined as the indications $x_{\mathrm{b}}$ and $y_{\mathrm{b}}$. If no torsion compensation was applied, then, after the wire had been moved from the idle position, the measurement system would have indicated its displacement with the value of $x_{\mathrm{p}}$ and $y_{\mathrm{p}}$, which are significantly different from the actual displacement of $x$ and $y$. The use of an additional, third laser rangefinder in the horizontal axis gives the possibility of compensating the indications, so that, despite the torsion of the wire, the result is consistent with its actual displacement. For the presented geometrical layout, the dependence involving displacements in the horizontal axis $x$ is as follows:

$$
x=\frac{\frac{x_{1}+x_{2}}{2}-y_{\mathrm{p}} \cdot\left(\frac{x_{1}-x_{2}}{l_{\mathrm{b}}}\right)}{1+\left(\frac{x_{1}-x_{2}}{l_{\mathrm{b}}}\right)^{2}}
$$

For the vertical axis, the following formula has been derived: 


$$
y=y_{\mathrm{p}}+\left[\frac{\frac{x_{1}+x_{2}}{2}-y_{\mathrm{p}} \cdot\left(\frac{x_{1}-x_{2}}{l_{\mathrm{b}}}\right)}{1+\left(\frac{x_{1}-x_{2}}{l_{\mathrm{b}}}\right)^{2}}\right] \cdot\left(\frac{y_{1}-y_{2}}{l_{\mathrm{b}}}\right)
$$

where: $x_{1}$ - change of the indication of the first rangefinder measuring the displacement in the horizontal axis $x ; x_{2}-$ change of the indication of the second rangefinder measuring the displacement in the horizontal axis $x ; y_{\mathrm{p}}$ - the apparent displacement of the contact wire in the vertical axis $y$ (change of the indication of the rangefinder measuring the displacement in this axis); $1 \mathrm{~b}$ - the distance between the rays emitted by the rangefinders recording the displacement in the horizontal axis.

During measurements, the electrical signals from the three rangefinders were recorded using an oscilloscope and then processed in a spreadsheet, in order to obtain the desired measurement results. Displacements in the vertical axis were recorded by a SICK rangefinder type OD250-150P142, whose measuring range is 100 to 400 $\mathrm{mm}$. Two rangefinders type OD130-50P142, also made by SICK, with the measuring range from 80 to $180 \mathrm{~mm}$, worked in the horizontal axis. A Tektronix TDS 540 oscilloscope, whose recording speed was set at 400 samples per second, was used to record the results.

\section{Measurements performed with the use of a visual system}

A visual system based on a high-speed 2D imaging camera, together with a system for recording advanced analysis of the image was used for measurements. The configuration of the visual measurement stand is presented in Fig. 4.

For the spatial configuration of the stand shown in Fig. 4, the displacement of the contact wire in the horizontal axis $x$ is defined by the dependence:

$$
x=\frac{(k-F) \cdot x^{\prime} \cdot \cos \beta}{\cos \alpha \cdot\left(F \cdot \cos \beta-y^{\prime} \cdot \sin \beta\right)-x^{\prime} \cdot \sin \alpha}
$$

Similarly, for the vertical axis $y$ the formula is:

$$
y=\frac{(k-F) \cdot\left(y^{\prime}+x^{\prime} \cdot \sin \beta \cdot \tan \alpha\right)}{F \cdot \cos \beta-y^{\prime} \cdot \sin \beta-x^{\prime} \cdot \tan \alpha}
$$

The measurements were performed with the use of Basler's 2D imaging camera, type acA 2040-180 kc, with the following basic technical specifications:

- sensor resolution: 2046x2046 px (4 Mpix);

- sensor size: $11.26 \times 11.26 \mathrm{~mm}$;

- maximum recording speed: $180 \mathrm{fps}$.

The camera was equipped with a Jupiter 9 2/85 lens, whose rated focal length is $f=84.944 \pm 0.078 \mathrm{~mm}$ (all the uncertainties presented in the article are standard uncertainties).

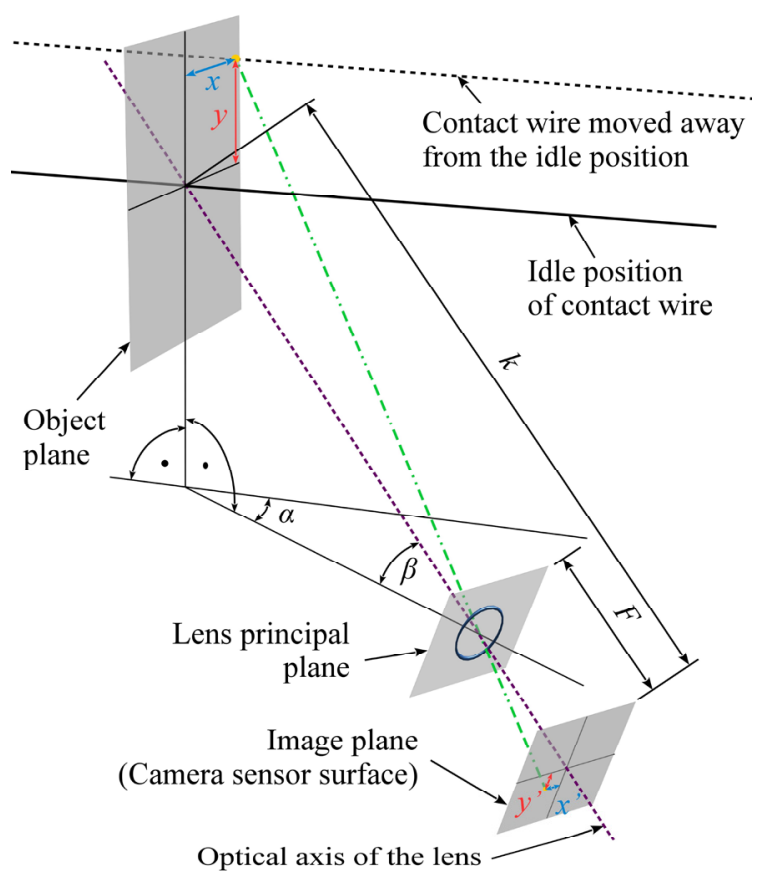

Fig. 4. Compensation of torsional vibrations with the use of a third laser rangefinder Configuration of the measurement stand, where: $x, y$-displacement of the contact wire in the horizontal and vertical axis respectively; $x^{\prime}, y^{\prime}$ - displacement of the image of the object on the camera sensor in the horizontal and vertical axis respectively, $k$ - the distance between the central point of the object plane and the image plane, $F$ - the distance between the lens principal plane and the camera sensor (plane of the image); $\alpha$ - the angle between the vertical plane in which the contact wire lies and the vertical plane in which the optical axis of the lens lies; $\beta$ - the angle of inclination of the optical axis of the lens in relation to the level.

The measurements were performed for the following stand configuration:

- distance $k=2742.46 \pm 0.24 \mathrm{~mm}$;

- $\quad$ distance $F=87.751 \pm 0.083 \mathrm{~mm}$;

- angle value for $\alpha=52.750 \pm 0.029^{\circ}$;

- $\quad$ angle value for $\beta=26.80 \pm 0.28^{\circ}$.

The measurements were performed at the maximum recording speed of the camera, which is slightly higher than the declared one, i.e. $187 \mathrm{fps}$. The recorded images were subjected to advanced processing in LabView in order to obtain the measurement results for $x^{\prime}$ and $y^{\prime}$, based on which, in accordance with the dependencies (3) and (4), the target measurement result, i.e. the displacement of the contact wire in the horizontal and vertical axis, was determined.

\section{Measurements results}

\subsection{Comparative measurements for contact wire oscillation}

A fragment of an exemplary course of displacement of the contact wire of the catenary network in the horizontal axis $x$ is shown in Fig. 5. Similar measurement performed for displacements in the vertical axis $y$ are shown in Fig. 6. 


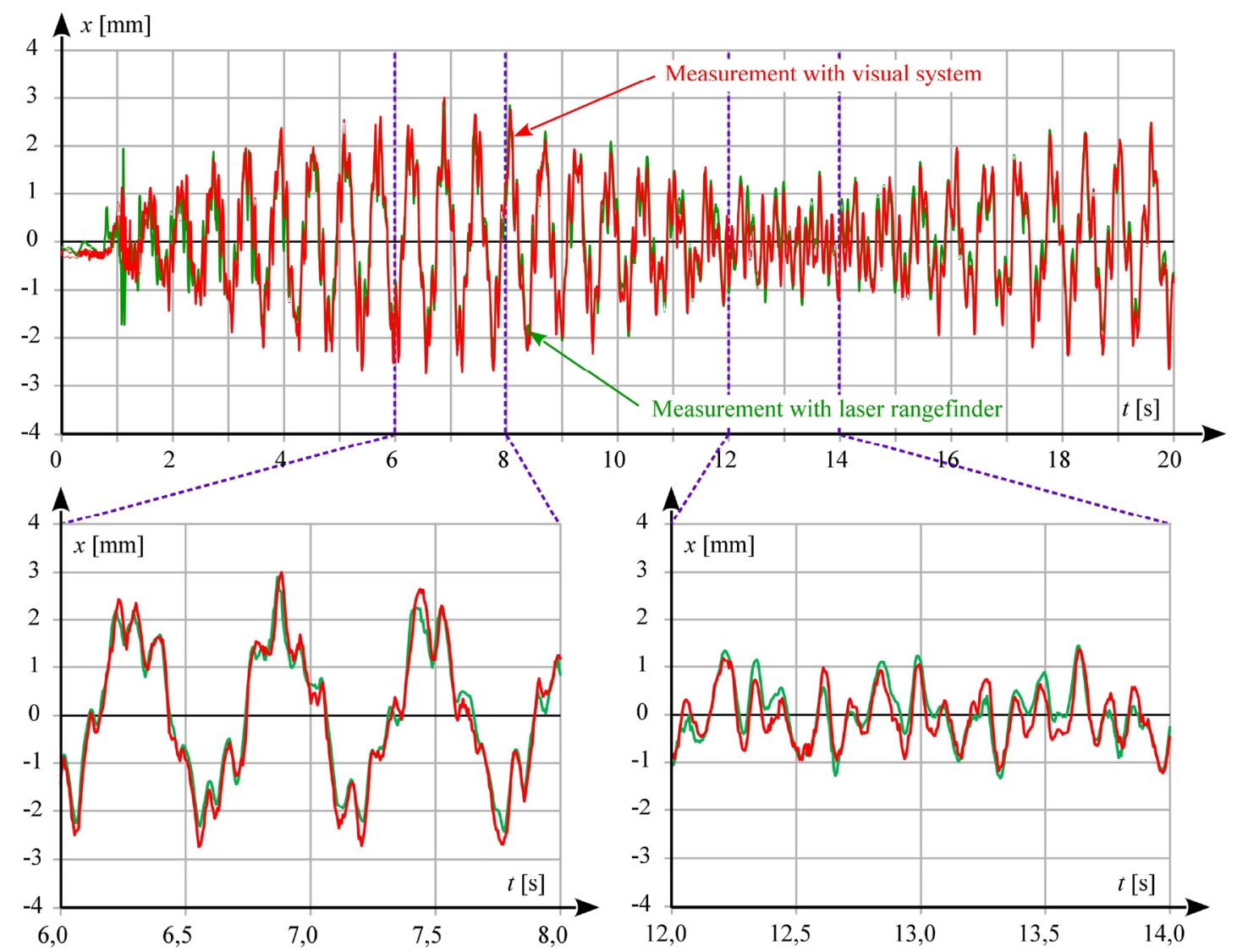

Fig. 5. Comparison of the results of measurements of the catenary network contact wire displacement in the horizontal axis $x$, performed with a laser rangefinder system and a visual system.

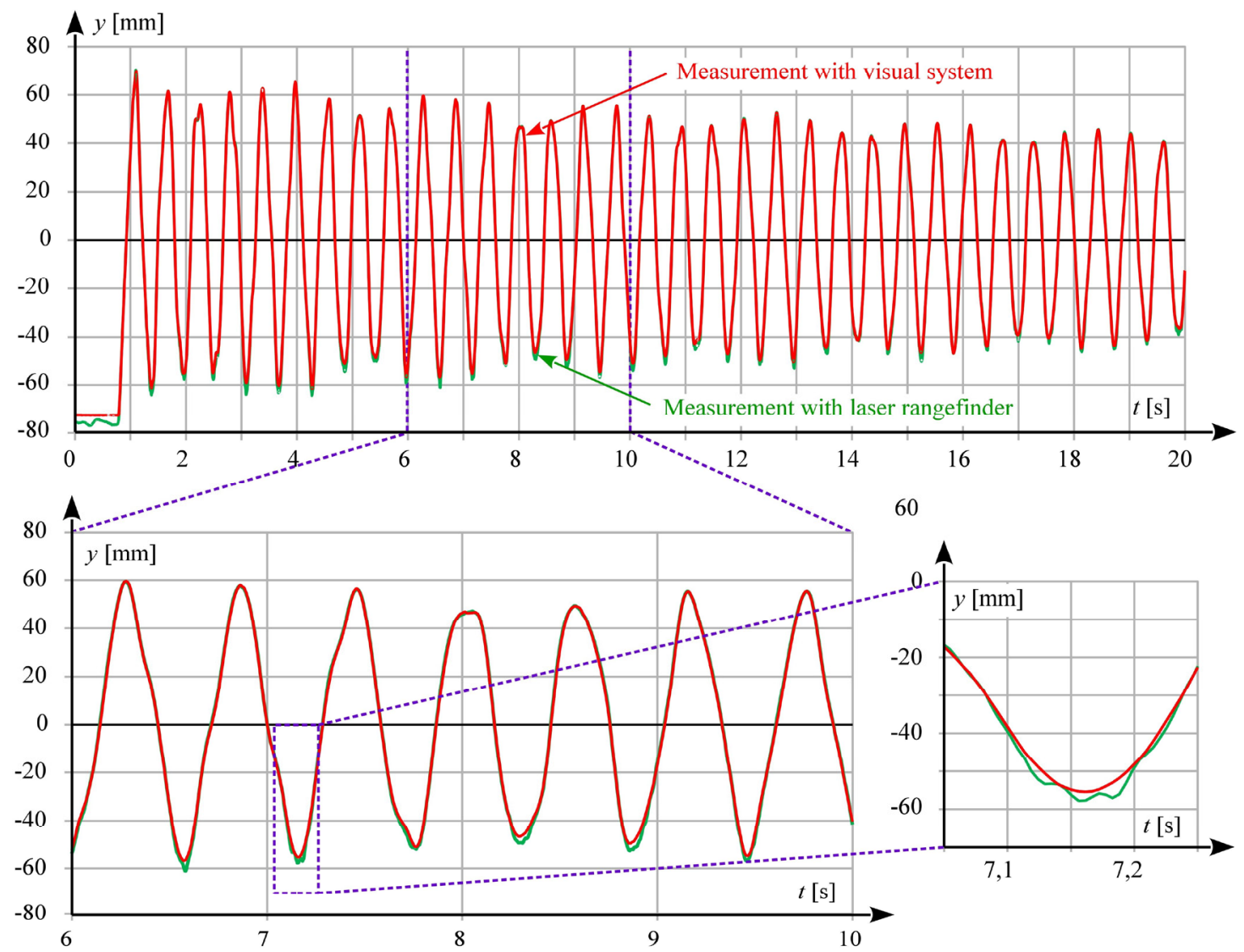

Fig. 6. Comparison of the results of measurements of the catenary network contact wire displacement in the vertical axis $y$, performed with a laser rangefinder system and a visual system. 
The measurement results show good convergence of the measurement results obtained with the use of both methods. For measurements of displacements in the horizontal axis, the differences in instantaneous values practically do not exceed $0.5 \mathrm{~mm}$ (the mean squared normalised error MSE is equal 0,048 mm), and the nature of the vibrations is reproduced in the same way in both cases. In the case of measurement of displacements in the vertical axis, there are differences of several millimetres in the results - MSE is equal 2,3 $\mathrm{mm}$ (see Fig. 6-enlargement), visible in the lower folding of the oscillation waveforms. This difference is caused by the mechanical characteristics of the reflective plates attached to the contact wire, which began to vibrate slightly in this position. It is visible in Fig. 6, where it can be observed that the trajectory of the measurement performed with the use of the visual system is smooth and the laser measurement is subject to additional oscillations.

\subsection{Comparative measurements for contact wire oscillation}

The measurement results presented in p. 6.1. show good convergence of the results obtained with both measurement methods and thus demonstrate the suitability of the two methods for performing the said measurements. However, they do not provide an answer to the question which method is more accurate, i.e. which results have a lower level of measurement uncertainty. To answer this question, measurements were performed at the stand shown in Fig. 2.

\subsubsection{Calliper reference measurement}

The result obtained with the use of a calliper was considered to be the reference measurement. For the calliper measurement, the value of the contact wire displacement is given by the formula:

$$
y_{\mathrm{pw}}=y_{\mathrm{w}}-D
$$

where: $y_{\mathrm{w}}$ - the distance between the contact wire vertical movement limiters; $D$ - wire diameter.

Values $y_{\mathrm{w}}$ and the diameter of the contact wire $D$ were measured using a Yato YT-7201 digital calliper with calibration uncertainty of $0.02 \mathrm{~mm}$ for the measurements below $100 \mathrm{~mm}$ and $0.03 \mathrm{~mm}$ for larger distances. A series of 10 measurements was performed for each of the values.

\subsubsection{Measurement performed with the use of a laser rangefinder}

For the measurements performed with a laser rangefinder the same rangefinder was used as in the measurement of oscillation courses, i.e. the OD250$150 \mathrm{P} 142$ model made by SICK. The current source of the rangefinder was connected to a reference resistor with the resistance of $R=100 \Omega$. The voltage signal was recorded by a Hioki recorder, model 8853 . The whole procedure involved the performance of 10 series of measurements. 500 measurements at the top position of the wire and then 500 measurements at its bottom position were recorded in each series. After averaging and calculating the difference, the displacement value was obtained for the given series. An example measurement is shown in Fig. 7.

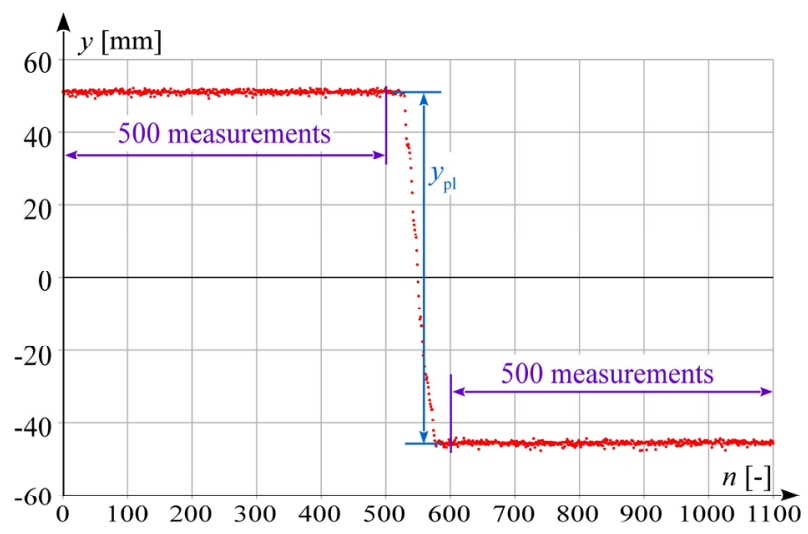

Fig. 7. Exemplary measurement performed with a laser rangefinder, where: $n$ - number of measurements.

\subsubsection{Measurement performed with the use of a visual system}

The analysis of measurement uncertainty showed that for visual measurements with the spatial configuration given in Fig. 4, the highest accuracy (the lowest level of measurement uncertainty) was obtained for the distance $k \approx 15 \mathrm{~m}$ [16]. To maintain the reproduction scale, a Sonnar 4/300 lens with a 2x type K-6B teleconverter was attached to the camera. The total rated focal length of such an optical system was $f=559.12 \pm 0.22 \mathrm{~mm}$. For such an optical system the other characteristics were:

- distance $k=14549.46 \pm 0.87 \mathrm{~mm}$;

- distance $F=582.43 \pm 0.23 \mathrm{~mm}$;

- $\quad$ angle $\alpha=42.7200 \pm 0.0057^{\circ}$;

- angle $\beta=5.290 \pm 0.067^{\circ}$.

For visual measurements, 10 measurement series were also performed, in an identical manner as for the laser rangefinder.

\subsubsection{Comparison of measurement results}

The results for a series of 10 measurements, along with standard uncertainties, are presented in Table 1. Due to multiple measurements, both type A and B uncertainties were taken into account.

Table 1. Comparison of measurement results.

\begin{tabular}{|c|c|c|}
\hline $\begin{array}{c}\text { Calliper } \\
\text { measurement } y_{\mathrm{ps}} \\
{[\mathrm{mm}]}\end{array}$ & $\begin{array}{c}\text { Laser rangefinder } \\
\text { measurement } y_{\mathrm{pl}} \\
{[\mathrm{mm}]}\end{array}$ & $\begin{array}{c}\text { Visual system } \\
\text { measurement } y_{\mathrm{pk}} \\
{[\mathrm{mm}]}\end{array}$ \\
\hline $97.245 \pm 0.023$ & $96.7 \pm 9.9$ & $97.206 \pm 0.066$ \\
\hline
\end{tabular}

As it can be seen, the differences in the results between the measurements performed with the laser rangefinder and the visual system, as well as the reference measurement, fall within the limits of measurement uncertainties. However, the considerably 
higher level of measurement uncertainty obtained when using a laser rangefinder is an interesting issue. It is a direct result of the manufacturer's declared uncertainty of calibration of the instrument, amounting to $\pm 3 \%$ of the maximum distance of the measurement scope, which gives the calibration uncertainty of $\Delta y=12 \mathrm{~mm}$ for the rangefinder used. The level of uncertainty obtained with visual measurements is three times higher than that of the reference measurement performed with the use of a calliper, and two orders of magnitude smaller than in the case of a laser rangefinder.

\section{Summary and conclusions}

The conducted experiment has proved the suitability of the visual measurement system for the analysis of vibrations and displacements of the contact line contact wire, or of its other elements. The obtained results are identical in quality to the existing ones, coming from a short range laser rangefinder measuring system, yet they are far superior in terms of accuracy. When using the visual system, the measurement uncertainty is two orders of magnitude smaller than in the case of laser rangefinders. Such a difference is a completely new measurement quality, unattainable when using the methods applied so far. Furthermore, the measurement of displacements in the bi-axial plane for the realization variant with the rangefinders is possible only in tests conducted in laboratory conditions. For field measurements on the catenary line, the laser rangefinders can only be used for measuring vertical displacements, which greatly limits the functionality of such measurements. Measurements of horizontal dislocations are practically impossible due to the difficulty in mounting the reflective plate and the rangefinders in such a way that they do not interfere with the current collector gauge. Such limitations do not occur during measurements performed with the visual system. Furthermore, the visual system does not require any additional elements to be mounted on the contact wire, so the mechanical parameters of the network itself are not changed. There are also no problems with simultaneous measurement of the displacements of the contact wire and the catenary, or both wires and the catenary, which, in the case of laser rangefinders, requires doubling or tripling the number of sensors. The disadvantages of the visual system include higher costs, the need for very precise positioning, and greater sensitivity to changes in the level of external lighting. However, as has been shown by the results of the analysis, in situations where high measurement accuracy is required, the visual system is irreplaceable.

\section{References}

1. Z. Giętkowski, K. Karwowski, M. Mizan, Diagnostics of contact line. (Wydawnictwo PG, Gdańsk, 2009) [In Polish]

2. S. Kusumi, K. Nezu, H. Nagasawa, Overhead Contact Line Inspection System by Rail-and-Road Car. RTRI Vol. 41 (4), 169-172 (2000)
3. Rav.M. Galiulin, Rish.M. Galiulin, J.M. Bakirov, V.V. Bydanov, D.R. Bogdanov, Optoelectronic measurement of a contact net geometric parameters in railways. IFAC Proceedings Volumes, Volume 33, Issue 20, 143-147 (2000)

4. H. Hofler, M. Dambacher, N. Dimopoulos, V. Jetter, Monitoring and inspecting overhead wires and supporting structures. IEEE Intelligent Vehicles Symposium/Parma, 512-517 (2004)

5. Z. Liu, W. Liu, Z. Han, A High-Precision Detection Approach for Catenary Geometry Parameters of Electrical Railway. IEEE Trans. Instrum. Meas., 111 (2017)

6. J. Skibicki, M. Bartłomiejczyk, Analysis of measurement uncertainty for contact-less method used to measure the position of catenary contact wire, performed with the use of Monte Carlo method. Measurement 97, 203-217 (2017)

7. I. Aydin, M. Karaköse, E. Akin, A robust anomaly detection in pantograph-catenary system Based on Mean-Shift Tracking and Foreground Detection. IEEE International Conference on Systems, Man, and Cybernetics. 4444-4449 (2013)

8. C.J. Cho, Y. Park, New monitoring technologies for overhead contact line at $400 \mathrm{~km} \cdot \mathrm{h}^{-1}$. Engineering 2, 360-365 (2016)

9. Z. Giętkowski, K. Karwowski, M. Mizan, P. Pazdro, Review of research and developement on the technical diagnostics in the field of electric traction. Pomiary Automatyka Kontrola 12, 66-70 (2003)

10. L. Jarzebowicz, S. Judek, 3D machine vision system for inspection on contact strips in railway vehicle current collectors. International Conference on Applied Electronics (AE). (2014)

11. S. Judek, L. Jarzebowicz, Algorithm for automatic wear estimation of railway contact strips based on 3D scanning results. International Conference and Exposition on Electrical and Power Engineering (EPE 2014). 724-729 (2014)

12. S. Judek, L. Jarzebowicz, Wavelet transform-based approach to defect identification in railway carbon contact strips. Elektronika ir Elektrotechnika, Vol. 21, No 6, 29-33 (2015)

13. E.D. Stefano, E. Ruffaldi, C.A. Avizzano, Automatic 2D-3D vision based assessment of the attitude of a train pantograph. IEEE International Smart Cities Conference (ISC2), 1-5 (2016)

14. K. Karwowski, M. Mizan, D. Karkosiński, Monitoring of current collectors on the railway line. Transport 0, 1-9 (2016)

15. C.A.L. Vázquez, M.M. Quintas, M.M. Romera, Non contact sensor for monitoring catenary-pantograph interaction. IEEE International Symposium on Industrial Electronics. 482-487 (2010)

16. J. Skibicki, The issue of uncertainty of visual measurement techniques for long distance measurements based on the example of applying electric traction elements in diagnostics and monitoring. Measurement 113, 10-21 (2017) 